\title{
Information Exchange rather than Topology Awareness: Cooperation between P2P Overlay and Traffic Engineering
}

\author{
Jia Zhao, ${ }^{1}$ Jianfeng Guan, ${ }^{2}$ Changqiao $\mathrm{Xu},{ }^{2}$ Wei Su, ${ }^{1}$ and Hongke Zhang ${ }^{1}$ \\ ${ }^{1}$ National Engineering Laboratory for Next Generation Internet Interconnection Devices, Beijing Jiaotong University, \\ Beijing 100044, China \\ ${ }^{2}$ State Key Laboratory of Networking and Switching Technology, Beijing University of Posts and Telecommunications, Beijing, China
}

Correspondence should be addressed to Jia Zhao; 11111004@bjtu.edu.cn

Received 3 November 2013; Revised 26 February 2014; Accepted 13 March 2014; Published 3 April 2014

Academic Editor: Yang Shi

Copyright ( 2014 Jia Zhao et al. This is an open access article distributed under the Creative Commons Attribution License, which permits unrestricted use, distribution, and reproduction in any medium, provided the original work is properly cited.

\begin{abstract}
Solutions to the routing strategic conflict between noncooperative P2P overlay and ISP underlay go separate ways: hyperselfishness and cooperation. Unpredictable (possibly adverse) impact of the hyperselfish topology awareness, which is adopted in both overlay routing and traffic engineering, has not been sufficiently studied in the literature. Topology-related information exchange in a cooperatively efficient way should be highlighted to alleviate the cross-layer conflict. In this paper, we first illustrate the hyperselfish weakness with two dynamic noncooperative game models in which hyperselfish overlay or underlay has to accept a suboptimal profit. Then we build a synergistic cost-saving (SC) game model to reduce the negative effects of noncooperation. In the SC model, through information exchange, that is, the classified path-delay metrics for $\mathrm{P} 2 \mathrm{P}$ overlay and peer locations for underlay, $\mathrm{P} 2 \mathrm{P}$ overlay selects proximity as well as saving traffic transit cost for underlay, and ISP underlay adjusts routing to optimize network cost as well as indicating short delay paths for P2P. Simulations based on the real and generated topologies validate cost improvement by SC model and find a proper remote threshold value to limit P2P traffic from remote area, cross-AS, or cross-ISP.
\end{abstract}

\section{Introduction}

P2P overlay systems impose tremendous traffic load on the Internet. Traffic engineering (TE) has been employed by Internet service providers (ISPs) to improve traffic transit cost on physical networks. Although P2P system and TE independently decide their own routing in different layers, they impact on each other, because routing strategic conflict exists between them. As shown in Figure 1, P2P overlay generates traffic with a profit objective to minimize all endto-end delays, while underlay TE adjusts its routing strategy to minimize network cost. Both peers $j$ and $k$ in overlay have the content that peer $i$ wants. Selfishly, peer $i$ chooses $k$. Unfortunately, underlay uses physical path CEFA to transit traffic $t_{k i}$ and cost (hops) two times more than path $B A$ from $j$ to $i$. Even if $i$ chose $j$, considering the background traffic in link $B A$, too much traffic volume from $j$ to a greedy peer $i$ would be a congestion risk for underlay network. Such crosslayer conflict has been analyzed in the literature [1-6].
Negative impacts on both overlay and underlay, resulted from noncooperation, can be summarized in three aspects. (i) Different profit objectives lead to selfish routing and may increase cost of each other, for example, increasing delay for P2P and maximum link utilization (MLU) for ISP. (ii) Underlay always has slow reaction to overlay traffic change, while overlay is prone to inaccurate proximity discovery. (iii) Every time when routing changes in overlay/underlay, it takes the two-layer system a period of time to readjust and converge to a new stable equilibrium, before which the system performs unstable oscillation.

Solutions for the noncooperative interaction above go separate ways as below.

(i) Hyperselfishness: more selfish than the noncooperation, self-improvement of underlay/overlay by detecting and benefiting from topology-related information of overlay/underlay without support of each other and regardless of possible profit damage to each other. 


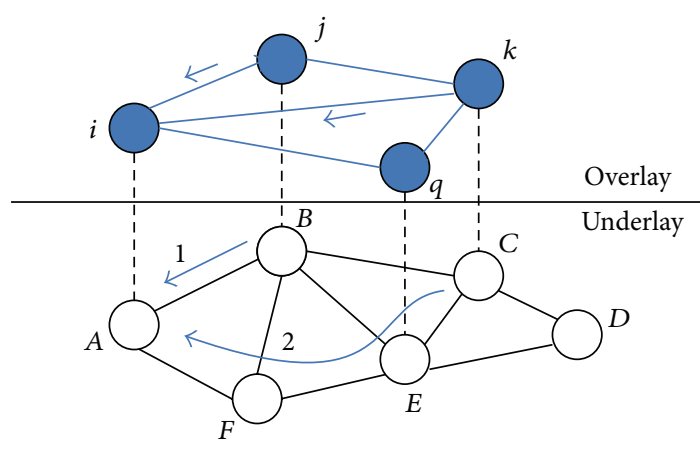

FIGURE 1: Overlay and underlay routing.

(ii) Cooperation: topology information exchange between cooperative overlay and underlay.

On the involvement of hyperselfishness, P2P overlays deploy network-aware function to make proximity selection [7-9], while ISPs upgrade traffic monitors and evolve multiprotocol label switching- (MPLS-) based TE or IP-based TE like equal-cost multipath (ECMP) [10-13]. On the involvement of cooperation, topology-related information services, such as Oracle and $\mathrm{P} 4 \mathrm{P}$, have been proposed and implemented [14-17]. Comparing the two ways, an inevitable problem for hyperselfish topology-awareness is the unpredictable (possibly adverse) impact on the topology-transparent one (i.e., underlay/overlay whose topology-related information is detected and known to overlay/underlay). Network-aware overlay may disarrange underlying global traffic optimization and peer-location-aware underlay may intercept and restrain overlying content-sharing flexibility. Accordingly, we have two goals in this paper: (i) to illustrate weakness of hyperselfish behaviors ans (ii) to utilize cooperation control to improve the three negative results from noncooperation.

ISPs and P2P content providers (CPs) always try selfimprovement unilaterally. In many practical scenarios, CP and ISP regard each other as opponent and rely much on their own technology to obtain opponent information (topology) for their selfish profit optimization. This hyperselfishness may intensify the "arm race" between CP and ISP [18]. Additionally, negative impact and inefficiency of hyperselfish topology-awareness has not been sufficiently studied in the literature. Hence, avoiding hyperselfish weakness will help CP and ISP to converge to cooperation of their own accord.

P2P overlays desire end-to-end path information to find proximity and minimize delay. ISPs pursue peer locations in $\mathrm{P} 2 \mathrm{P}$ to adjust routing and save traffic transit cost. Therefore, it is reasonable for a P2P system and an ISP to cooperate to exchange information and embody a common goal in their respective profit functions. In contrast to selfish profit loss, the common goal to reciprocate each other is a way to a winwin situation [18].

In this paper, our main contributions include the following. (1) We illustrate hyperselfish weakness with two dynamic noncooperative game models. In the dynamic games, overlay/underlay initiates hyperselfish topology-awareness, and then topology-transparent underlay/overlay uses a reacting strategy as self-protection or punishment to the hyperselfish one. We explain why hyperselfish initiator, though having opponent information may not benefit more and have to bow to a suboptimal profit. (2) We build a synergistic cost-saving (SC) game model based on topology-related information exchange between underlay and overlay. On one hand, ISPs indicate a classified path metric (e.g., delay, distance, or other link cost metrics) for P2P to select proximity and generate traffic demand rationally. A remote threshold (RT) is also used to limit P2P traffic from remote area, cross-AS, or cross-ISP. On the other hand, underlay obtains peer-location information from CPs and chooses physical paths to split traffic for optimal network cost, for example, minimum MLU. Due to the cooperation above, the overlay chooses proximity as well as saving transit cost for the underlay, while the underlay decides routing to optimize network cost as well as indicating short delay path for the overlay. Simulation results validate the cost improvement by SC model and the importance of proper RT selection.

The remainder of the paper is organized as follows. Section 2 introduces related work. In Section 3, we use two dynamic game models to illustrate hyperselfish weakness. In Section 4, we propose the SC model and detail the mechanisms of path classes and RT selection. Simulations are shown in Section 5. Section 6 concludes the paper.

\section{Related Work}

Cross-layer conflict and negative results from noncooperation have been researched in the literature. Liu et al. first model the interaction between overlay routing and TE with a noncooperative game [1]. They consider delay as cost for both the layers and show that selfish overlay routing may cause huge cost and oscillations to the two-layer system. In the paper [3], the authors model the dynamic games in which the leader layer has preemptive strategy to restrain routing readjustment of the follower layer so as to overcome negative impact of the performance oscillations. Wang et al. study the noncooperation between P2P overlay and TE and propose that the interaction impacts the two layers and causes a nonoptimal performance of both layers [4].

Solutions to the conflict are also proposed in related work. DiPalantino and Johari use the congestion signal, matched to TE objective, to obtain efficient equilibrium in the game between TE and content distribution [2]. With regard to cooperation in papers $[14,15]$, physical path distances are provided for $\mathrm{P} 2 \mathrm{P}$ overlay to select closer peers and save network cost. Jiang et al. propose a game with sharing control to save costs for cooperative CPs and ISPs [16]. For P2P traffic management, bilateral cooperation between network operator and peers of P2P systems is proposed in [17]. In the paper [18], authors analyze the cross-layer interaction pattern and give an overview of information that can be exchanged between cooperative P2P systems and ISPs.

One main difference between our work and the related work above lies in that we use two dynamic game models to analyze the hyperselfish behaviors of overlay/underlay who unilaterally tries topology-awareness to profit from topology 
information of the other one, regardless of possible damage to each other. With emergence of more and more unilateral improvement such as evolving TE or network-aware P2P systems, it is necessary to prompt ISPs and P2P systems to avoid such possibly adverse topology-awareness and use cooperation to solve cross-layer conflict. Our work also differs from $[2,16]$ in that the cooperation way we use is the exchanged information including peer-locations and pathdelay metrics. Additionally, differing from [14, 15, 17], our paper proposes path classes and remote threshold with which ISPs and P2P systems can achieve cost improvement with a common goal to limit traffic from remote areas.

\section{Weakness of Hyperselfishness}

Diversity of topology-aware mechanisms has been used as solution to the cross-layer conflict between P2P overlay routing and ISP's traffic engineering. These mechanisms are hyperselfish because either overlay or underlay only consider its own optimal routing and accordingly cannot deal with the possible adverse effect which causes damages to both layers. In this section, we build two dynamic game models to demonstrate the weakness of hyperselfish topologyawareness, and we use these models to explain the limitation of self-improvement from either layer.

3.1. Negative Impacts of Noncooperation. Both P2P overlay and ISP underlay have their own routing strategies and utility objectives. Peers in a P2P system demand contents from closer peers to obtain QoS, for example, fast file-download and fluent video playback $[19,20]$. The utility of P2P overlay is to minimize total end-to-end delays. Underlay use TE and selects paths to transit traffic. The utility of the underlay is to optimize the overall network cost.

Overlay and underlay independently decide their strategy, but the strategy they choose will influence the utility of each other. Accordingly, a noncooperative game model can be built for interaction between overlay and underlay [21]. In a network $G=(V, E)$ and its overlay node set $N$ and given the notations and descriptions in Notations, we model the noncooperative game as follows.

Underlay chooses the routing strategy $R=\left\{r_{i j}^{k}(e) \cdot f_{i j}^{k}\right\}$ by solving the problem as follows:

$$
\min G\left(R, M_{T}\right)=\sum_{e \in E i, j \in N} \sum_{k \in S_{i j}} g_{e}\left(t_{i j}, r_{i j}^{k}(e) \cdot f_{i j}^{k}\right)
$$

subject to $\sum_{k} f_{i j}^{k}=1$, for all $e \in E, S_{i j}=\left\{1, \ldots,\left|p_{i j}\right|\right\}, p_{i j}=$ $\left\{p_{i j}^{k}\right\}, t_{e}^{b}+\sum_{k} \sum_{i, j} t_{i j} \cdot r_{i j}^{k}(e) \cdot f_{i j}^{k} \leq c_{e}$.

Overlay decides the overlay traffic matrix $M_{T}=\left\{t_{i j}\right\}$ by solving the problem as follows:

$$
\left.\min H\left(M_{T}, R\right)=\sum_{e \in E i, j \in N} \sum_{i j} h_{i j}, P\left(p_{i j}\right)\right)
$$

subject to $t_{e}^{b}+\sum_{i, j \in N} \sum_{k \in S_{i j}} t_{i j} \cdot r_{i j}^{k}(e) \cdot f_{i j}^{k} \leq c_{e}, \sum_{i \in N} t_{i j}=T_{j}$.

Function $g_{e}(\cdot)$ represents the transit cost function of link $e, G\left(R, M_{T}\right)$ is underlay's total cost function whose value is decided by $R$ and $M_{T}$, function $h_{i j}(\cdot)$ represents the delay from overlay node $i$ to $j, H\left(M_{T}, R\right)$ is overlay's total cost function whose value is decided by $R$ and $M_{T},\left|p_{i j}\right|$ denotes number of elements in physical path set $p_{i j}$ for $i j$, and $P\left(p_{i j}\right)$ denotes the underlay routing policy for logical link $i j$.

From this basic model, we draw three negative results from the cross-layer conflict.

3.1.1. Selfish Routing Conflict. Different utilities may lead to conflicting routing strategies. P2P system select closer peers to minimize delay. When the physical path under the logical link between two peers is overloaded, this shortest-delay choice of overlay may be a congestion risk to underlay links. On the other side, because underlay uses TE to optimize global traffic transit regardless of QoS of P2P overlay, TE routing adjustment may map overlay logical link between geographically nearby peers into a long-delay physical path.

3.1.2. Inefficiently Detecting Each Other. ISPs always deploy infrastructures to monitor traffic and update a traffic matrix periodically, so as to make optimal routing selection. Yet, overlay is always active and the traffic changes frequently. So, underlay routing is not instantly adjusted and subject to time lag. Besides, inefficient network-aware machinery of P2P overlay may cause inaccurate proximity selection.

3.1.3. Slow Convergence and Oscillation. Although it can be proved that strategy equilibrium exists in the game, the actual converging process will experience several rounds of adjustments from both overlay and underlay. During these rounds, selfish and rational overlay/underlay will react to the other by changing routing to obtain optimal utility again and again. Frequent readjustment (oscillation) and longtime of equilibrium convergence will bring systematic instability.

3.2. Hyperselfish Weakness of Overlay. We regard overlay and underlay as two players in the dynamic game. Unlike simultaneous strategy decision of noncooperative game in Section 3.1, one player in the dynamic game decides his strategy before the other one decides his, and the later player has the information of the first's choice [22]. Hyperselfish overlay as the later player tries to use network-awareness to obtain underlay routing information and make selfish profit optimization regardless of possible profit damage to underlay. As the first player, underlay has the advantage of knowing overlay's strategic rule which underlay can use to decide an optimal reacting strategy to restrain overlay profit to be suboptimal. To analyze weakness of overlay hyperselfish behavior, we model the dynamic game as follows.

According to the detected underlay strategy $R$, overlay chooses optimal traffic matrix as follows:

$$
M_{T}^{*}(R)=\arg \min H\left(M_{T}, R\right) .
$$

Underlay punishes the hyperselfish behavior $M_{T}^{*}(R)$ with an optimal reacting routing strategy as follows:

$$
R^{*}=\arg \min G\left(M_{T}^{*}(R), R\right)
$$


subject to $\sum_{k} f_{i j}^{k *}=1, S_{i j}^{*}=\left\{1, \ldots,\left|p_{i j}^{*}\right|\right\}, p_{i j}^{*}=\left\{p_{i j}^{k *}\right\}$, for all $e \in E, t_{e}^{b}+\sum_{i, j \in N} \sum_{k \in S_{i j}^{*}} t_{i j}^{*} \cdot r_{i j}^{k *}(e) \cdot f_{i j}^{k *} \leq c_{e}, \sum_{i \in N} t_{i j}^{*}=T_{j}$, $i, j \in N$.

In this game, hyperselfish overlay obeys the strategic rule $M_{T}^{*}(R)$ to generate as much as possible traffic $M_{T}^{*}$ via the detected short-delay paths from network-awareness result $R$. This behavior is a congestion risk and disarranges underlying global traffic optimization. To restrain this hyperselfishness, underlay can use (4) to first choose action $R^{*}$ as selfprotection before overlay's possible adverse action $M_{T}^{*}(R)$.

Proposition 1. A subgame perfect information Nash Equilibrium solution exists in the dynamic game about overlay hyperselfish behavior, if link cost function $g_{e}$ and overly delay cost function $h_{i j}$ are all continuous.

Proof. On any link $e$, overlay traffic is subject to $t_{\text {over }}^{e} \leq c_{e}$. There are strategic constrains of $\sum_{k} f_{i j}^{k}=1$ and $\sum_{i \in N} t_{i j}=T_{j}$. So, solution set $S=\left\{\left(M_{T}, R\right)\right\}$ is a closed space. Because $g_{e}$ and $h_{i j}$ are continuous, continuous function gets minimal value at the solution $R^{*}$, and $H$ gets the minimal value at $M_{T}^{*}$. We have the inequalities as $G\left(M_{T}^{*}, R^{*}\right) \leq G\left(M_{T}^{*}, R\right)$ and $H\left(M_{T}^{*}, R^{*}\right) \leq H\left(M_{T}, R^{*}\right)$. When overlay decides the policy $M_{T}^{*}$ and underlay decides the policy $R^{*}$, they will not deviate from this equilibrium solution, because they cannot gain more profit with other policies. Therefore, $\left(M_{T}^{*}, R^{*}\right)$ is a subgame perfect information Nash Equilibrium.

Proposition 2. Overlay's hyperselfish behavior and underlay's reaction can be modeled as the Stackelberg competition.

Stackelberg's leadership model [23] is a game between two players; one of which is the leader and decides his strategy first and the other one is the follower and decides his strategy after the leader's decision. In the competition between overlay and underlay, underlay is the leader who first deploys traffic engineering in its networks to limit the traffic from selfish overlay routing.

Proposition 3. Overlay hyperselfish behavior can just obtain a suboptimal profit when underlay decides an optimal reacting strategy to restrain the overlay.

Proof. Underlay takes the leadership to control the traffic through its networks. The control strategies are decided to limit some unfriendly traffic that cause high transit cost and to ensure QoS of ISP-friendly traffic. P2P traffic and background traffic (from ISP-friendly applications) will compete for network bandwidth. The dynamic game can be formatted as follows:

$$
\begin{aligned}
& t_{i j}^{*}\left(P\left(p_{i j}\right)\right)=\arg \max \sum_{P\left(p_{i j}\right)} t_{i j}, \\
& P^{*}\left(p_{i j}\right)=\arg \min \sum_{e \in E} \sum_{i, j \in N} \sum_{k \in S} w_{e} \cdot t_{i j}^{*}\left(P\left(p_{i j}\right)\right) \cdot r_{i j}^{k}(e) \cdot f_{i j}^{k} .
\end{aligned}
$$

Hyperselfish P2P overlay obeys the rule $t_{i j}^{*}\left(P\left(p_{i j}\right)\right)$ to generate as much as possible traffic on its detected short-delay

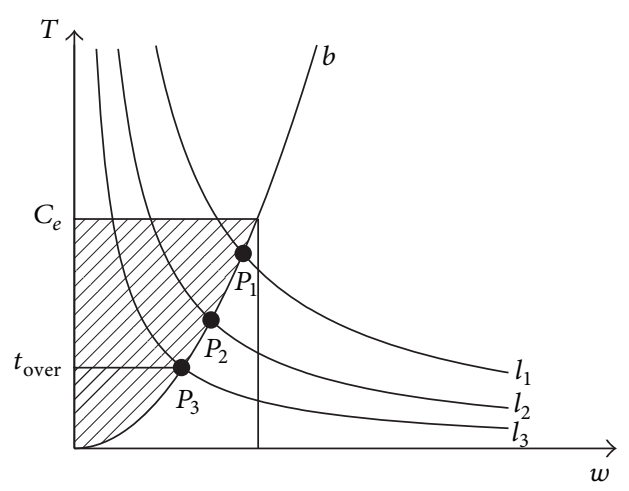

FIGURE 2: Suboptimal profit for hyperselfish overlay.

path $p_{i j}$. Underlay uses $P^{*}\left(p_{i j}\right)$ as a congestion-proof strategy to reserve some bandwidth, leave enough bandwidth for ISPfriendly traffic, and leave the limited bandwidth for P2P traffic on the path $p_{i j}$.

Considering each single link, we have the link cost $l=$ $t_{e} \cdot w_{e}$. As shown in Figure $2, l_{1}, l_{2}$, and $l_{3}$ are three different equal-cost curves. Vertical axis indicates traffic amounts $t_{e}$. Horizontal axis indicates $w_{e}$, that is, link cost per traffic unit. Suppose that the link capacity is $C_{e}$. Curve $b$ represents $\mathrm{P} 2 \mathrm{P}$ traffic up-bound limited by ISP at each link (across horizontal $W$-axis). Curve $b$ is an increasing function because we want $t_{\text {under }}=C_{e}-b\left(w_{e}\right)$ to be the bandwidth for ISP-friendly traffic. The cheaper (e.g., shorter delay or lower congestion) the link is, the more ISP-friendly traffic the link has, so that it ensures QoS of ISP-friendly traffic. According to (6), the underlay's strategy is to obtain the minimum link cost $l=$ $t_{e} \cdot w_{e}$. From the three equal cost curves, underlay will choose $l_{3}$. According to (5), overlay will choose $t_{\text {over }}$ from the three points $P_{1}, P_{2}$, and $P_{3}$ on curve $b$ because they are the maximum traffic volumes the overlay can generate. According to Proposition 2, underlay is the leader in this game and first chooses $l_{3}$. Then overlay follows underlay's decision to choose the value $t_{\text {over }}$ in Figure 2 . Hence, cross-point $P_{3}$ is the equilibrium point of overlay and underlay. Compared with $P_{1}$ and $P_{2}, \mathrm{P} 2 \mathrm{P}$ overlay traffic $t_{\text {over }}$ at equilibrium point $P_{3}$ is a suboptimal traffic.

For example in Figure 1, peer $i$ chooses peer $j$ rather than peer $k$ to generate traffic demand, because overlay detects that underlay uses shorter (one hop) path 1 to transit traffic from $j$ to $i$. If ISP limits $\mathrm{P} 2 \mathrm{P}$ traffic on path $B A$, the low available bandwidth for $\mathrm{P} 2 \mathrm{P}$ traffic on this path may also cause long time overlay delay. In this case, P2P's networkaware machinery is of no avail.

Take the interaction between MPLS-TE and networkaware P2P as an example. Suppose that a P2P system deploys the topology-aware algorithm and accordingly find the shortest delay path from requester to responder. The requesting peer decides to demand traffic via this detected-best-path and desires download speed as fast as possible. So, profit utility of the requester is to maximize his available download link bandwidth. What the P2P overlay does above is hyperselfish. This unilateral behavior may cause troubles (e.g., congestion 
or bandwidth shortage) for the underlay ISPs when other background traffic also request bandwidth resource. Once the ISP discovers this hyperselfish intention, MPLS-TE can be used as self-adjustment for underlay and punishment to overlay. MPLS-TE enables ISP to map different services to multiple label switched paths (LSPs). Assisted by resource reservation protocol (RSVP), MPLS will provide background traffic (other ISP-friendly applications) with reserved path bandwidth firstly and optimally. Then, even if a short-delay end-to-end is detected by P2P, much bandwidth resource on this detected path has been reserved by ISP for transit cost-saving, leaving limited available bandwidth for P2P. This limitation by underlay will impact the QoS for overlay peers, but overlay cannot change this suboptimal profit by itself.

3.3. Hyperselfish Weakness of Underlay. Hyperselfish underlay regards $\mathrm{P} 2 \mathrm{P}$ overlay as opponent and detects $\mathrm{P} 2 \mathrm{P}$ traffic volume through physical network. As the first player, knowing underlay's strategic rule, overlay can firstly choose an optimal reacting strategy before underlay's action. The dynamic game is formatted as follows,

According to detected overlay traffic matrix $M_{T}$, underlay chooses optimal routing strategy as follows:

$$
R^{*}\left(M_{T}\right)=\arg \min G\left(M_{T}, R\right) \text {. }
$$

Overlay reacts to the hyperselfish behavior $R^{*}\left(M_{T}\right)$ with an optimal traffic decision as follows:

$$
M_{T}^{*}=\arg \min H\left(M_{T}, R^{*}\left(M_{T}\right)\right)
$$

subject to $\sum_{k} f_{i j}^{k *}=1, S_{i j}^{*}=\left\{1, \ldots,\left|p_{i j}^{*}\right|\right\}, p_{i j}^{*}=\left\{p_{i j}^{k *}\right\}$, for all $e \in E, t_{e}^{b}+\sum_{i, j \in N} \sum_{k \in S_{i j}^{*}} t_{i j}^{*} \cdot r_{i j}^{k *}(e) \cdot f_{i j}^{k *} \leq c_{e}, \sum_{i \in N} t_{i j}^{*}=T_{j}$, $i, j \in N$.

Proposition 4. A subgame perfect information Nash Equilibrium solution exists in the dynamic game about underlay hyperselfish behavior, if link cost function $g_{e}$ and path delay cost function $h_{i j}$ are all continuous.

This dynamic game model is applicable to many practical scenarios, where ISPs depend on traffic monitors to obtain traffic statistics of $\mathrm{P} 2 \mathrm{P}$ or other applications. The statistics are used for bandwidth demand prediction or traffic routing adjustment in TE. Take bandwidth prediction as an example. ISPs always depend on current traffic state to predict bandwidth demand in future. ISP's TE wants to reserve optimal bandwidth for other ISP-friendly application traffic. So, TE control the underlay-friendly traffic $t_{u}^{e}$ on each link $e$, while $\mathrm{P} 2 \mathrm{P}$ overlay control the $\mathrm{P} 2 \mathrm{P}$ traffic $t_{o}^{e}$ on each link. Then underlay traffic and P2P traffic will compete for surplus bandwidth. The bandwidth competition game can be expressed as follows.

To find the optimal strategy $t_{u}^{e}$, underlay solves the following problem:

$$
\max \frac{\left(\sum_{e} c_{e}-\sum_{e} t_{u}^{e}-\sum_{e} t_{o}^{e}\right) \cdot\left(\sum_{e} t_{u}^{e}\right)}{T} .
$$

To find the optimal strategy $t_{o}^{e}$, overlay solves the following problem:

$$
\max \frac{\left(\sum_{e} c_{e}-\sum_{e} t_{u}^{e}-\sum_{e} t_{o}^{e}\right) \cdot\left(\sum_{e} t_{o}^{e}\right)}{T}
$$

subject to $t_{u}^{e}+t_{o}^{e} \leq c_{e}, \sum_{e} t_{u}^{e}+\sum_{e} t_{o}^{e} \leq T$.

$c_{e}$ denotes the capacity of link $e$. Constant $T$ is the limited total traffic volume. TE controls the incremental ISP-friendly traffic to take up the surplus bandwidth, which is matched with the current proportion of $t_{u}^{e}$ to the total traffic amount T. P2P overlay also wants its incremental bandwidth matched with the current P2P traffic proportion $\left(\sum_{e} t_{o}^{e}\right) / T$. If TE tries to decide $t_{u}^{e}$ according to $t_{o}^{e}$ which is detected by the ISP traffic monitor, overlay can first decide a strategy $t_{o}^{e *}$ as selfprotection according to underlay's strategic rule $t_{u}^{e *}\left(t_{o}^{e}\right)$.

Proposition 5. Hyperselfish underlay has a suboptimal result in the bandwidth competition with P2P overlay.

Proof. Considering each link, underlay decides $t_{u}^{e}$ and overlay decides $t_{o}^{e}$. We compare the two games: noncooperation and hyperselfishness. The former is a Cournot model [24] and can be expressed as follows,

Underlay solves the problem: $\max \left(c_{e}-t_{u}^{e}-t_{o}^{e}\right) \cdot t_{u}^{e} / T$. Overlay solves the problem: $\max \left(c_{e}-t_{u}^{e}-t_{o}^{e}\right) \cdot t_{o}^{e} / T$.

Solutions are $t_{u}^{e *}=t_{o}^{e *}=c_{e} / 3$. Both underlay's and overlay's bandwidth profit equal $c_{e}^{2} / 9 T$.

The dynamic game of hyperselfishness is a Stackelberg model [23] as follows:

$$
\begin{aligned}
t_{u}^{e *}\left(t_{o}^{e}\right) & =\arg \max \left(c_{e}-t_{u}^{e}-t_{o}^{e}\right) \cdot \frac{t_{u}^{e}}{T}=\frac{\left(c_{e}-t_{o}^{e}\right)}{2}, \\
t_{o}^{e *} & =\arg \max \left(c_{e}-t_{u}^{e *}\left(t_{o}^{e}\right)-t_{o}^{e}\right) \cdot \frac{t_{o}^{e}}{T}=\frac{c_{e}}{2} .
\end{aligned}
$$

Underlay profit equals $c_{e}^{2} / 16 T$, and overlay profit equals $c_{e}^{2} / 8 T$.

Comparing the results in the two cases above, optimal bandwidth profit in hyperselfish case is inferior to optimal result in noncooperative case. So, hyperselfish underlay profit is suboptimal.

Besides, hyperselfish weakness of underlay can also be ascribed to the routing change with $\mathrm{P} 2 \mathrm{P}$ traffic state. ISPs rely too much on traffic monitoring infrastructures and distribute traffic engineering protocols (e.g., MPLS, ECMP) in many network nodes. So, it takes a relatively long time to update traffic statistics and to converge distributed TE to a stable equilibrium every time when overlay traffic change happens. Such hypersensitivity but slow reaction of the underlay fails to control the frequently changing P2P traffic.

3.4. From Hyperselfishness to Cooperation. Self-improvement by either CPs or ISPs may not achieve efficiency, though much effort has been made to evolve machinery for either traffic control or network-awareness. On the one hand, ISPs are committed to upgrade TE and enhance traffic control. MPLS-based TE can label different protocol packets and 
establish tunnels for differentiated traffic. Changeable IGP link weights or BGP routing attributes are deployed for intra- or interdomain TE, for example, SculpTE [12]. Due to efficient traffic monitoring technologies [10], virtual network topology based TE systems, such as AMPLE, are proposed to split traffic and balance load [25]. By central routing control for software defined network (SDN), MPLS-TE on OpenFlow platform is implemented [11]. Yet, evolving TE will still encounter routing conflict with overlay because $\mathrm{P} 2 \mathrm{P}$ traffic changes are random. On the other hand, P2P systems keep on improving proximity discovery or network-aware mechanism (e.g., TopBT, CLOSER, and NAPA-WINE) and overlay structure (e.g., tree, mesh, and DHT) to select shortdelay paths and satisfy traffic demand among peers efficiently [7-9]. Nevertheless, inaccurate and unreliable delay measurement and poor topology-aware performance will still be the bottleneck in overlay utility optimization.

In many scenarios, without cooperation, no matter how much effort ISPs or CPs make, there is just a suboptimal profit for them. Hyperselfish topology-awareness does not solve the cross-layer routing problem because the path information which either layer gets by topology-awareness is not accurate or in real time. Such topology-awareness may cause damage to both layers. Hence, it is reasonable for both layers to cooperate by a deliberate mechanism of information exchange.

\section{A Synergistic Cost-Saving Game}

In contrast with noncooperation and hyperselfishness, cooperation enables underlay and overlay to optimize selfish profit without damaging each other. We highlight a common goal in cost functions of both underlay and overlay. Advantage of the common goal is due to reciprocity, that is, self-optimization as well as considering and supporting profit goal of the other. Topology-related information can be exchanged between cooperative underlay and overlay to achieve the common goal. With peer locations, for example, IP addresses, from overlay, underlay can indicate path delay between overlay peers. With path information, for example, delay metric such as IGP weight, from underlay, overlay can select closer peers to avoid generating traffic from remote area, cross AS, or cross ISPs.

According to Propositions 1 and 4 , the link cost function $g_{e}$ and the overly delay cost function $h_{i j}$ should be continuous. Let $g_{e}(x)=u_{i j}^{e} \cdot x$ and let $h_{i j}(x)=v_{i j} \cdot x$ be two linear functions. These continuous and convex functions on the strategy set ensure the existence of the Nash Equilibrium solution. Our synergistic cost-saving game is modeled as follows.

Underlay chooses $R=\left\{r_{i j}^{k}(e) \cdot f_{i j}^{k}\right\}$ by solving the problem:

$$
\min \sum_{i, j \in N} \sum_{e \in E} \sum_{k \in S} u_{i j}^{e} \cdot\left(t_{i j} \cdot f_{i j}^{k} \cdot r_{i j}^{k}(e)+t_{b}^{e}\right) .
$$

Overlay decides $M_{T}=\left\{t_{i j}\right\}$ by solving the problem:

$$
\min \sum_{i, j \in N} v_{i j} \cdot t_{i j}
$$

subject to $\sum_{k} \sum_{i, j} t_{i j} \cdot f_{i j}^{k} \cdot r_{i j}^{k}(e)+t_{b}^{e} \leq c_{e}, \sum_{k} f_{i j}^{k}=1, \sum_{i} t_{i j}=T_{j}$. $v_{i j}$ refers to logical path-delay metric from $i$ to $j$ and is given by underlay. $u_{i j}^{e}$ refers to link cost to transit per unit traffic on physical link $e$ if $e$ is on logical path $i j$. Path metric set $\left\{v_{i j}, \forall i, j \in N, i \neq j\right\}$ for all logical peer connections are classified into numbers of path classes according to the path metric value. For example in Figure 1, we take hop-count as the path-delay metric. Underlay maps logical path $i j$ into physical path $A B$ (1 hop), $j k$ into $B C$ (1 hop), qk into $E C$ (1 hop), $i k$ into $A B C$ (2 hops), $q i$ into EFA (2 hops), and $k i$ into CEFA ( 3 hops). These paths can be classified into three classes as $\{i j, j k, q k\},\{i k, q i\}$, and $\{k i\}$.

Proposition 6. A Nash Equilibrium solution exists in the synergistic cost-saving game.

Proof. Link cost function in formula (12) and path-delay cost function in formula (13) are both continuous functions. Because of the constraints of $t_{\text {over }}^{e} \leq c_{e}, \sum_{k} f_{i j}^{k}=1$, and $\sum_{i \in N} t_{i j}=T_{j}$, solution set $S=\left\{\left(M_{T}, R\right)\right\}$ is a closed space. So, both underlay and overlay can get minimal costs at the solution $S^{*}=\left\{\left(M_{T}^{*}, R^{*}\right)\right\}$, which satisfies $\sum_{i, j \in N} \sum_{e \in E} \sum_{k \in S} u_{i j}^{e}$. $\left(t_{i j}^{*} \cdot f_{i j}^{k *} \cdot r_{i j}^{k *}(e)+t_{b}^{e}\right) \leq \sum_{i, j \in N} \sum_{e \in E} \sum_{k \in S} u_{i j}^{e} \cdot\left(t_{i j}^{*} \cdot f_{i j}^{k} \cdot r_{i j}^{k}(e)+t_{b}^{e}\right)$ and $\sum_{i, j \in N} v_{i j}^{*} \cdot t_{i j}^{*} \leq \sum_{i, j \in N} v_{i j}^{*} \cdot t_{i j}$. Underlay and overlay will not deviate from the solution $S^{*}=\left\{\left(M_{T}^{*}, R^{*}\right)\right\}$, because they cannot gain more profit with other policies. So, $S^{*}=$ $\left\{\left(M_{T}^{*}, R^{*}\right)\right\}$ is a Nash Equilibrium solution.

In this synergistic game model, mechanisms of path information, path classes, and remote threshold are detailed as follows.

4.1. Path Information. Because each link has a weight to indicate delay or other cost metrics, each underlying physical path, consisting of one or multiple links, can get an accumulated weight, which is sum of link weights on the path. For logical path $i j$ in overlay, underlay can split traffic into multiple physical paths. So, we use the average weight $w_{i j}$ of $k$ paths to express the path weight for $i j$ as follows:

$$
w_{i j}=\sum_{k} w_{i j}^{k} \cdot f_{i j}^{k} .
$$

The path metric $v_{i j}$ equals to or positively correlate to the path weight $w_{i j}$. According to the value of $v_{i j}$, path $i j$ will be classified into a path class and the class number $x_{i j}$ will be given to overlay. Because $v_{i j}$ is given by underlay, overlay has the accurate path-delay for proximity selection.

Obtaining the peer locations from P2P overlay, for each logical path between peers, ISP underlay can provide the path information which includes the following.

(i) Path class number $x_{i j}$ : indicating the path class which ij belongs to.

(ii) Remote threshold (RT) value $k$ : a selected class number, used to distinct between near and remote path classes.

(iii) Limited traffic volumes $T_{i j}: T_{i j}$ is given only when $x_{i j}>k$ (to limit traffic from remote candidate peer $i$ to requesting peer $j$ ). 
(iv) Path-delay metric $v_{i j}: v_{i j}$ is given only when $x_{i j} \leq k$. By solving formula (13), overlay can decide optimal traffic from $i$ to $j$.

For large-scale P2P overlays, it is difficult for ISP underlay to select a remote threshold from numerous logical paths, yet due to path classes, a proper class number can be selected as RT to limit traffic from remote areas and significantly improve cost for both overlay and underlay.

4.2. Remote Threshold. Remote threshold can be used to divide paths into the near and the remote. Suppose there are total $n$ path-classes in the network. Path metric values increase with order of class numbers. $x_{i j}$ is the class number of the logical path $i$ to $j$. The threshold value is set to be $k$. When underlay gets the peer couple of $i$ and $j$, it will calculate the path weight $w_{i j}$. Path $i j$ can be classified into one of the $n$ path classes. If the class number $x_{i j}$ which $i j$ belongs to is greater than $k$, distance between $i$ and $j$ is regarded as the remote, and the traffic from $i$ to $j$ is limited. If $x_{i j}>k$, path metric $v_{i j}$ is not shown to overlay and only a limited value of traffic volume is included in path information for overlay. If $x_{i j} \leq k, v_{i j}$ will be given to overlay, and overlay will use proximity optimal algorithm to generate traffic from the closer peers. This threshold mechanism helps overlay quickly exclude remote peers or generate traffic less than the limitation from remote peers.

ISPs do not want to transit cross-AS or cross-ISP traffic, because interdomain links cost much more than intradomain. In this scenario, RT can be used as a threshold for binary value classes, for example, " 0 " represents intradomain and " 1 " represents interdomain. When path class number $x=$ 1 , traffic on this path is limited. Through this way, underlay can guide overlay peers to generate traffic within the AS or the ISP.

4.3. Proximity Selection Process of Overlay. Only if two peers are close enough, that is, path class number not greater than RT, overlay can use the proximity selection algorithm to decide traffic between the two peers. Before making optimal traffic decision, overlay depends on the path information to delete remote candidate peers or know the traffic limitation from those peers.

Overlay solves the formula (13) to obtain optimal traffic demand. Before using the path metric $v_{i j}$, overlay has to consider path congestion risk and multiply $v_{i j}$ by a weight. The weight is related to traffic demand $t_{i j}$, because $t_{i j}$ affects the link delay. If link $e$ is on the path $i j$, link delay is expressed as

$$
D_{e}=\frac{1}{c_{e}-t_{i j}^{e}-t_{b}^{e}} .
$$

Too much traffic into link $e$ will cause long time delay. This part of delay cost should be included in the path weight.

For example in Figure 1, underlay uses path $B A$ to transit $t_{j i}$, path CEFA to transit $t_{k i}$, and path EFA to transit $t_{q i}$. Assume that total traffic demanded by $i$ is a constant $t$. We use hop-count to weight each path. So $v_{j i}=1, v_{q i}=2$, and

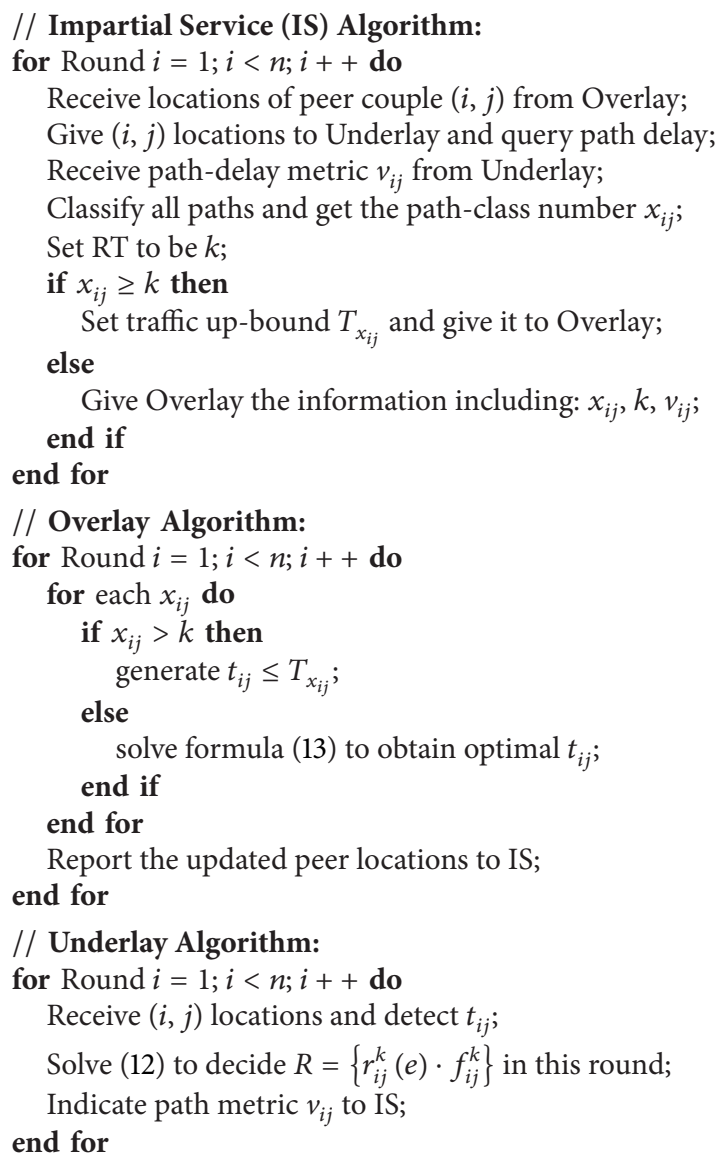

Algorithm 1: Synergistic cost-saving process.

$v_{k i}=3$. The remote threshold is set to be 2 . So, the overlay path $k i$ is deleted by $i$. Assume that the links of the underlay have the same capacity 1 . Multiplying the path weight by the delay factors, we get the new path weights as $v_{j i}=1 /\left(1-t_{j i}\right)$ and $v_{q i}=2 /\left(1-t_{q i}\right)$. With these new path weights, we solve the formula (13) as follows:

$$
\min \frac{t_{j i}}{1-t_{j i}}+\frac{2 t_{q i}}{1-t_{q i}}
$$

subject to $t_{j i}+t_{q i}=t, t_{j i} \geq 0, t_{q i} \geq 0$. So, peer $i$ can demand optimal traffic from $j$ and $q$.

4.4. Algorithm. Information can be directly exchanged between P2P system and ISP or through an impartial service between overlay and underlay. SC optimization is shown as Algorithm 1.

\section{Performance Evaluation}

In this section, based on simulation in both real and generated topologies, we validate that SC model improves the negative impacts of noncooperation and a proper remote threshold should be selected for cost improvement. 
5.1. Simulation Setup. To demonstrate the improvement for noncooperative negative impacts which have been summarized in Section 3, we use real network topology of Abilene [26] for analysis. Also, we use GT-ITM [27] to generate three network topologies for proper remote threshold selection. The backbone topology of Abilene has 11 point-of-presence nodes and its actual link capacities. End-to-end latencybased IGP link weights in [28] can be used to calculate the path metric $v_{i j}$ in our SC model. We set the background traffic by referring to traffic statistical analysis of Abilene [29]. We refer to [30] and generate overlay traffic on several logical overlay paths. GT-ITM can generate transit-stub style topologies which can simulate ISP networks. We generate three topologies, each of which consists of 5 transit nodes and 2 stub domains connected to each transit node. Every stub domain has 3 nodes on average. Totally, in each topology, we get 35 underlay nodes and set the link capacity between each two nodes to be $10 \mathrm{Mbps}$. In each topology, 10 underlay nodes are mapped into a fully connected 10-node overlay. Each overlay node has its own constant traffic demand from other overlay nodes. We use hop-count as the path metric $v_{i j}$ for overlay peering pairs. Then SC algorithm can be deployed for cost optimization. To select a proper RT from path classes for the three topologies, we use one topology for analytical selection and use the other two for validating rationality of the selected threshold. Besides, we add the delay factor as format of (15) to the path weights which are used in overlay proximity selection.

5.2. Improvement by SC. We use SC model with path classes to improve the negative impacts of noncooperation. In the game-theoretic optimization, underlay and overlay take several rounds to converge to a strategic equilibrium, that is, optimal traffic demand between peers for overlay and optimal physical path selection and traffic splits for underlay. In each round, according to the strategic decision of underlay/overlay, overlay/underlay will react and adjust with an optimal strategy by executing SC Algorithm. We study the case that each node in overlay has a constant total traffic demand from other nodes and decides fraction of traffic demand from every other node. This optimization takes several rounds because overlay needs to react to routing change of underlay in each round. On the other hand, underlay will react to traffic change between overlay peers in each round and make an optimal routing adjustment. Noncooperative game takes many rounds to converge to equilibrium and makes the two-layer system in unstable oscillation. In addition to slow convergence, suboptimal cost for both overlay and underlay leave margin for improvement. In contrast with noncooperation, our SC model uses topology-related information exchange to help both overlay and underlay to improve their suboptimal costs and shorten the convergence duration. We also investigate whether pathclass mechanism helps SC go further to save costs.

Overlay traffic is set to 50 percents of the total traffic volume with the network utilization of 0.4 in the Abilene topology. We compare three cases: noncooperation, SC with path class mechanism in optimal algorithm and SC without

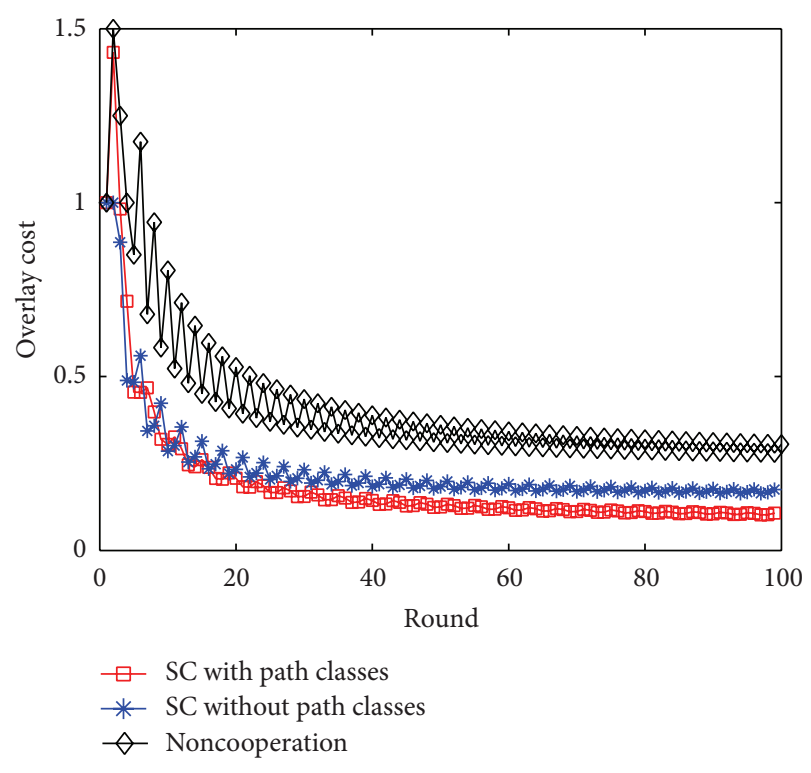

FIGURE 3: Overlay cost improvement.

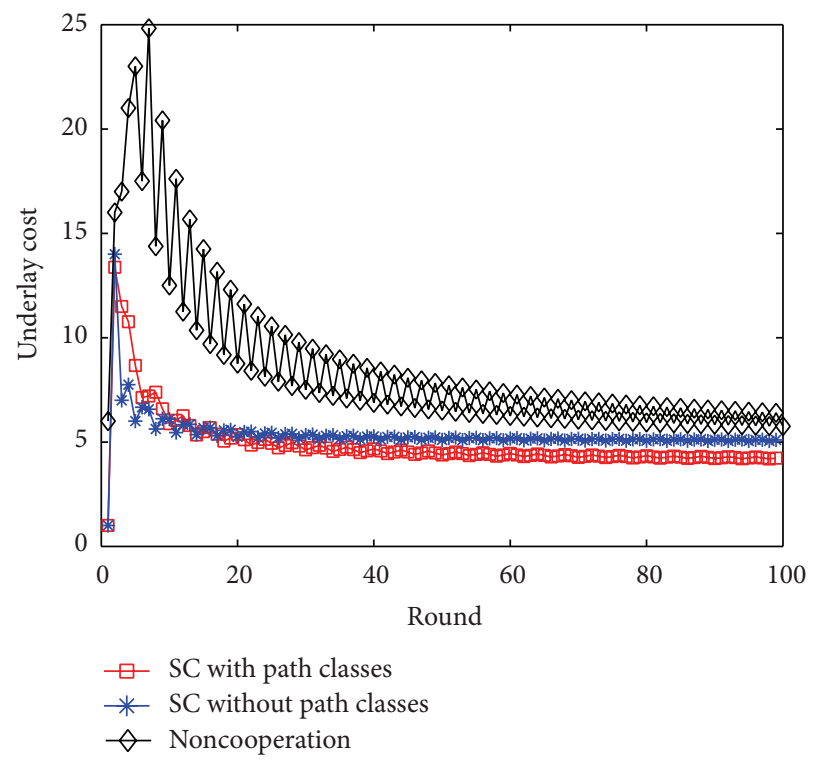

FIGURE 4: Underlay cost improvement.

path classes. Figures 3 and 4, respectively, show overlay cost (normalized average delay relative to the initial value 1) and underlay cost (normalized MLU relative to the initial value 1) in 100 rounds. We can see that two types of SC have a faster converging speed to equilibrium than noncooperation. Although the costs flap with independently reacting decisions of the two layers in different rounds, yet due to information exchange between cooperative overlay and underlay, SC shows far slighter oscillation than noncooperation case and obtains systematical stability. Comparing stable costs of SC with noncooperation, SC saves about $30 \%$ cost in overlay and about $20 \%$ cost in underlay. 


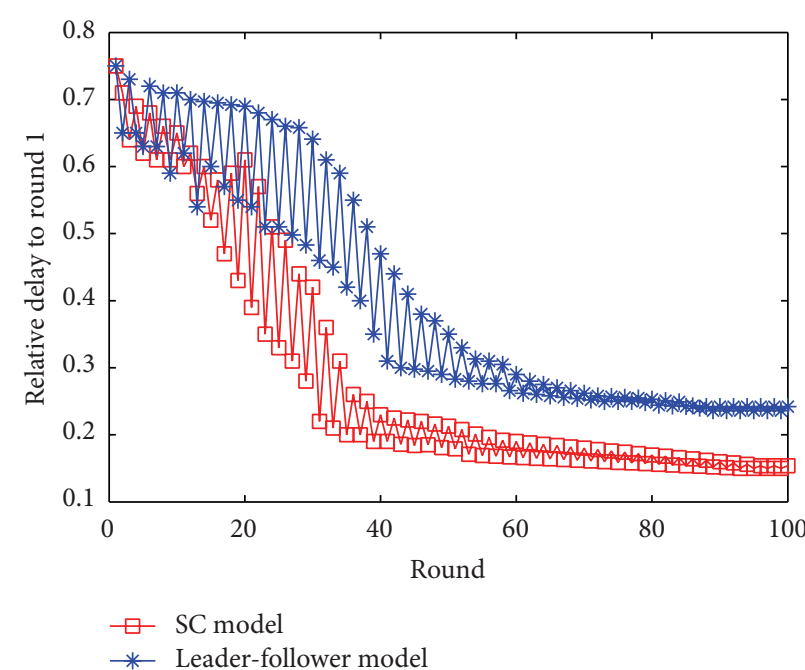

(a)

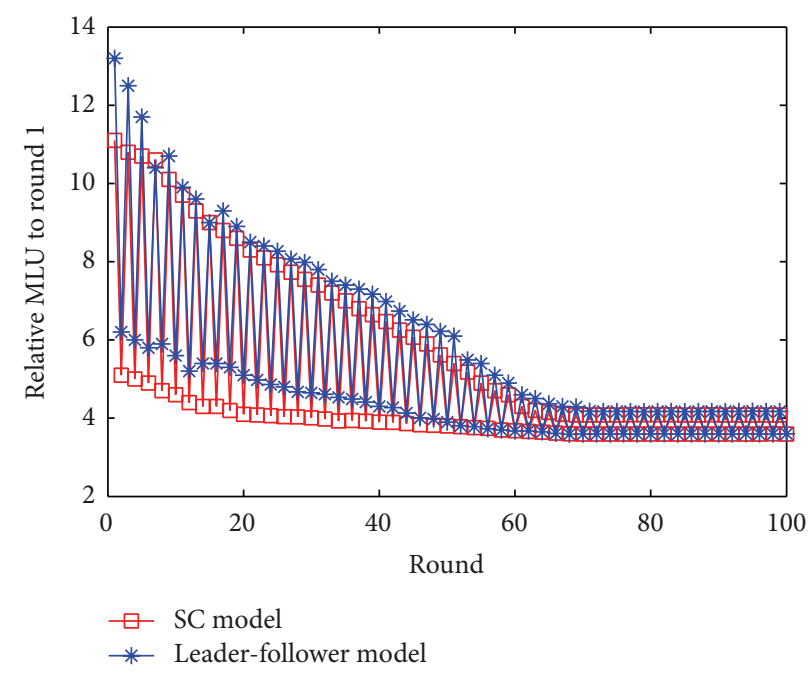

(c)

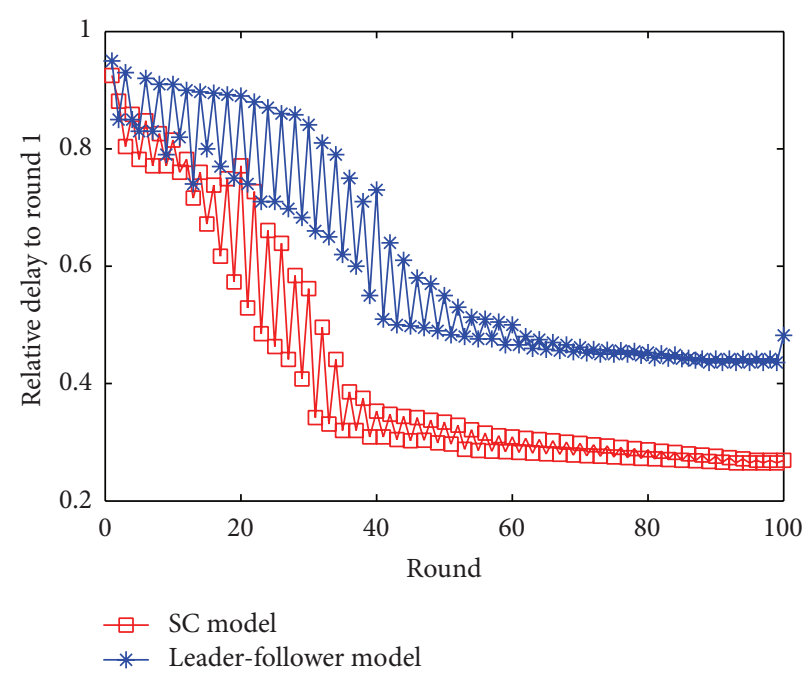

(b)

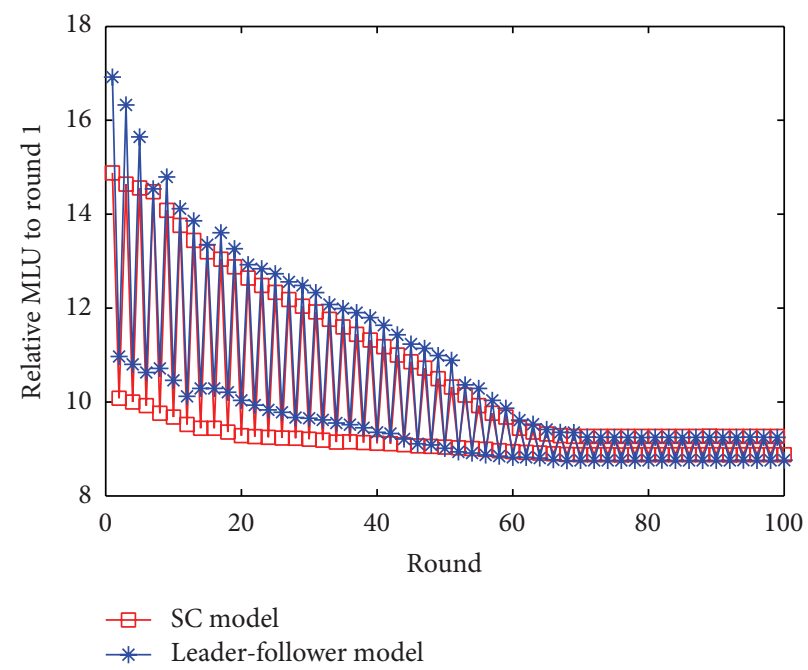

(d)

Figure 5: Comparison of two models at the overlay traffic proportion $\rho$ : (a) $\rho=30 \%$, (b) $\rho=60 \%$, (c) $\rho=30 \%$, and (d) $\rho=60 \%$.

It is also remarkable that $\mathrm{SC}$ with path classes outperforms SC without path classes. That is because classified path can help overlay node quickly delete remote candidate peers in order to minimize average end-to-end delay. Hence, both overlay and underlay take advantage of the common goal to limit traffic from remote areas. We also compare SC model with the leader-follower model [3] at different proportions of overlay traffic. As shown in Figure 5, though two models perform nearly the same in the underlay cost Figures 5(c) and 5(d), SC model performs much better than the other for saving overlay cost at the relatively high load of overlay traffic.

5.3. Proper RT Selection. We use a topology generated by GTITM to explore the existence of a proper remote threshold of path classes. Hop account on physical path is used as path metric $v_{i j}$ for overlay. We simulate performance of the two-layer system in 300 seconds and use cost results at four observation time points for analysis. In Figures 6(a) and 6(b), when remote threshold $k \leq 3$, the system shows lower and closer costs (delay and MLU) in stable state than $k=4$ case. Results demonstrate that if underlay does not limit traffic on the path whose class is larger than 3 , overlay and underlay costs will increase significantly. So, a proper threshold can distinct between near and remote so as to leave closer candidate peers for an overlay node to generate traffic demand optimally.

5.4. Rationality of Selected RT. We use the other two topologies generated by GT-ITM to validate rationality of the threshold selection. For each topology we set threshold $k$ to be 3 and simulate system performance 25 times. Convergence duration means how long time it takes to arrive at the state of cost equilibria (i.e., stable delay, and stable MLU). Totally, we get 50 experimental points from the two topologies. Results are shown in Figure 7. Most (about 80\%) of the points are around the point $(13,31,0.042)$ which is approximate to the 


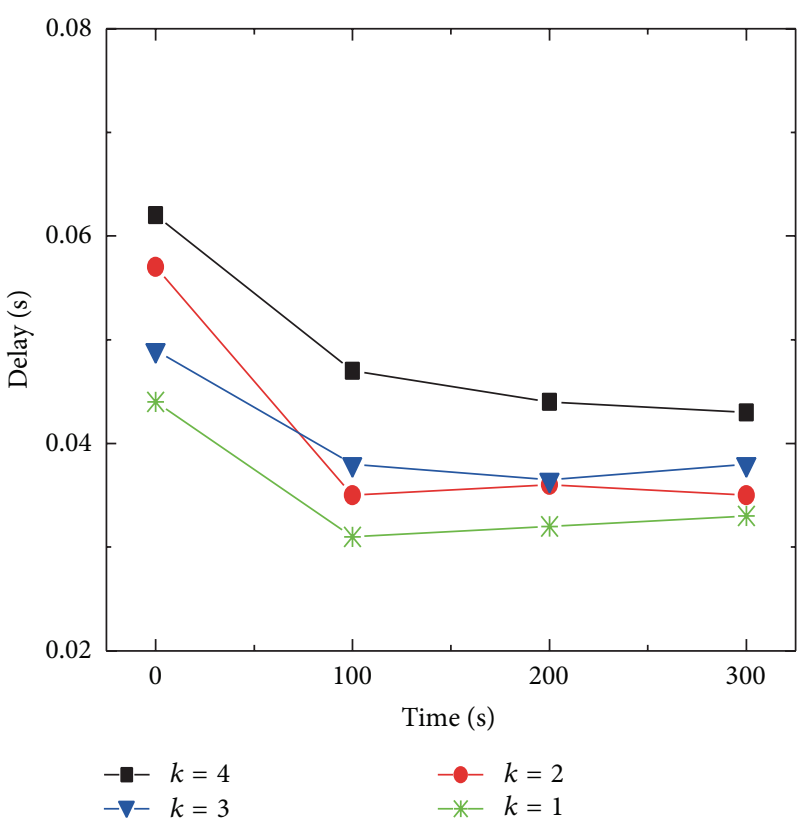

(a)

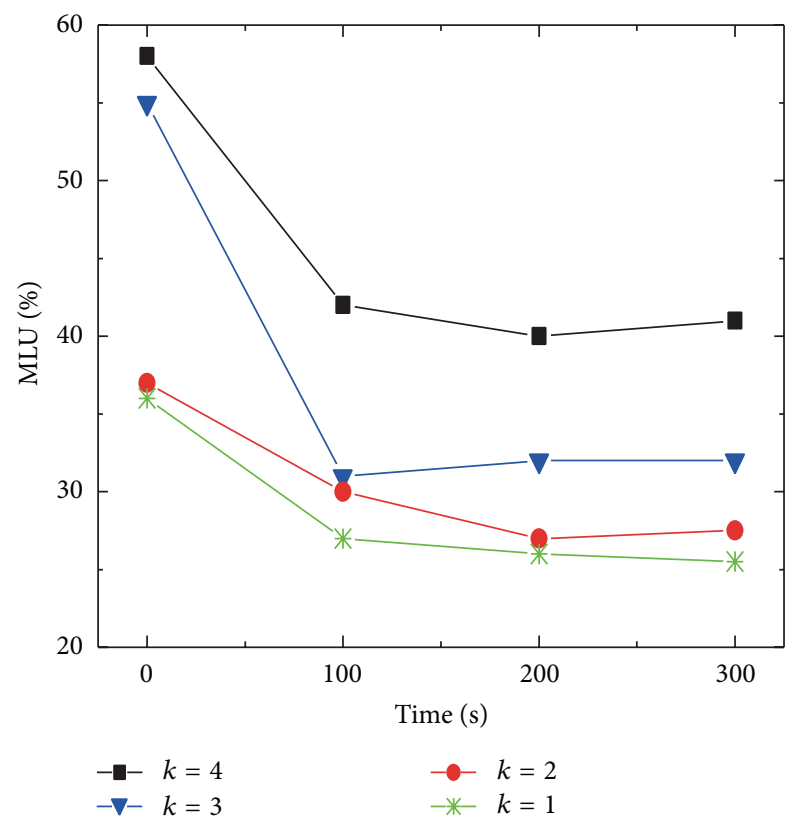

(b)

Figure 6: (a) Delay of overlay at four RT values. (b) MLU of underlay at four RT values.

average experimental result in Section 5.3. So, the threshold value we selected is applicable to the same size networks (transit-stub topology with 35 underlay nodes and 10 overlay nodes). Corresponding to a topology with SC solution, a proper remote threshold exists and should be selected for cost improvement. Accordingly, it is efficient and convenient for ISPs to select a proper RT for one network and apply the RT to other same scale networks.

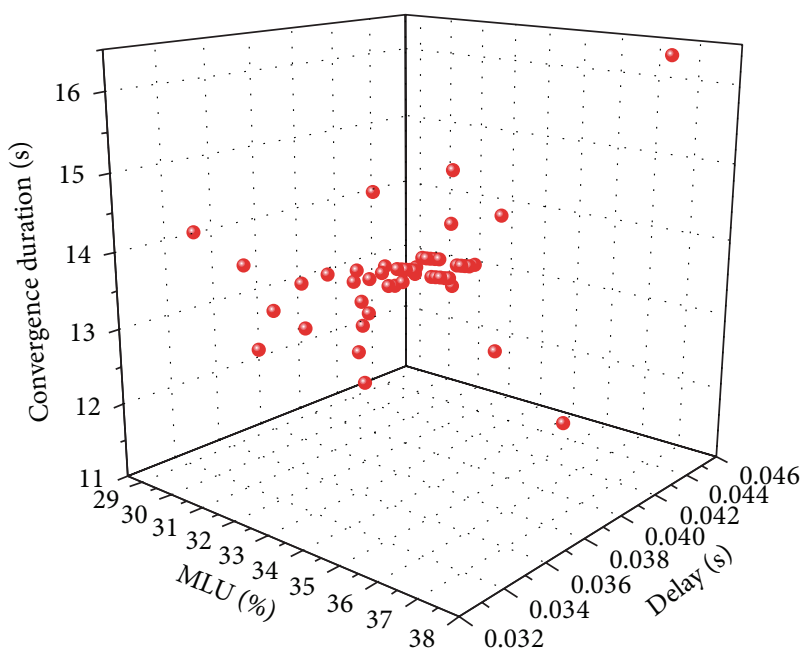

Figure 7: 50 times of test results when RT is equal to 3.

Path classes and RT are even more important for largescale ISP topologies. One of the main goals of ISP underlay in this cost-saving cooperation is to divide paths into near and remote and to limit traffic from remote areas. Path classes are helpful for this coarse-grained division because ISP can choose a proper RT from limited numbers of classes rather than find threshold value from all of the numerous paths. Mechanism of path class enables ISPs to select proper RT quickly and efficiently for saving cost of traffic transit.

\section{Conclusion}

In this paper we investigate cross-layer conflict between P2P overlay and ISP underlay and highlight solutions to use cooperation rather than hyperselfish topology-awareness. Two dynamic game models are built to illustrate suboptimal profit of hyperselfish initiator. We build a topology-informationexchange-based game model to improve negative impact of noncooperation. Simulation results validate that SC model with path classes can improve costs for both layers and decrease the time consumption to arrive at the stable equilibrium. The proper remote threshold selection is also proved essential for cost improvement of the two-layer system. With popularity of $\mathrm{P} 2 \mathrm{P}$ streaming and content oriented networks, our future work will pertain to ISP traffic management through cooperation with $\mathrm{P} 2 \mathrm{P}$ streaming systems and crossISP content distribution between cooperative ISPs.

\section{Notations}

$V:$ Node set of ISP's underlay network

$E$ : Set of links, $e \in E$, $e$ connects two underlay nodes

$N$ : Overlay node set, $i, j \in N$

$i j$ : Overlay logical link from $i$ to $j$

$p_{i j}^{k}$ : The $k$ th underlay routing path for logical path $i j$

$t_{i j}$ : Traffic amount from $i$ to $j$

$c_{e}$ : Capacity of link $e$

$t_{e}^{b}$ : Background traffic on link $e$ 
$T_{j}: \quad$ Total traffic demanded by overlay node $j$

$f_{i j}^{k}$ : Fraction of $t_{i j}$ on path $p_{i j}^{k}$

$r_{i j}^{k}(e)$ : Value 1 if link $e$ on path $p_{i j}^{k}$, and 0 otherwise

$v_{i j}$ : $\quad$ Metric of logical path delay from $i$ to $j$

$w_{e}$ : Cost of per traffic unit transit on link $e$.

\section{Conflict of Interests}

The authors declare that there is no conflict of interests regarding the publication of this paper.

\section{Acknowledgments}

This work is supported by the National Basic Research Program of China (973 Program) under Grant no. 2013CB329101 and by the 863 Program under Grant no. 2011AA010701 and by the National Natural Science Foundation of China (NSFC) under Grant nos. 61372112 and 61232017 and by the Beijing Natural Science Foundation (4142037).

\section{References}

[1] Y. Liu, H. Zhang, W. Gongt, and D. Towsley, "On the interaction between overlay routing and underlay routing," in Proceedings of the 24th Annual Joint Conference of the IEEE Computer and Communications Societies (INFOCOM '05), pp. 2543-2553, Miami, Fla, USA, March 2005.

[2] D. DiPalantino and R. Johari, "Traffic engineering vs. content distribution: a game theoretic perspective," in Proceedings of the 28th IEEE International Conference on Computer Communications (INFOCOM '09), pp. 540-548, Rio de Janeiro, Brazil, April 2009.

[3] S. Seetharaman, V. Hilt, M. Hofmann, and M. Ammar, "Resolving cross-layer conflict between overlay routing and traffic engineering," IEEE/ACM Transactions on Networking, vol. 17, no. 6, pp. 1964-1977, 2009.

[4] C. Wang, N. Wang, M. Howarth, and G. Pavlou, "On the interactions between non-cooperative P2P overlay and traffic engineering behaviors," in Proceedings of the 53rd IEEE Global Communications Conference (GLOBECOM '10), Miami, Fla, USA, December 2010.

[5] J. Kurian and K. Sarac, "A survey on the design, applications, and enhancements of application-layer overlay networks," $A C M$ Computing Surveys, vol. 43, no. 1, article 5, 34 pages, 2010.

[6] J. Dai, F. Liu, and B. Li, "The disparity between P2P overlays and ISP underlays: issues, existing solutions, and challenges," IEEE Network, vol. 24, no. 6, pp. 36-41, 2010.

[7] R. Birke, E. Leonardi, M. Mellia et al., "Architecture of a network-aware P2P-TV application: the NAPA-WINE approach," IEEE Communications Magazine, vol. 49, no. 6, pp. 154-163, 2011.

[8] M. P. Manzillo, L. Ciminiera, G. Marchetto, and F. Risso, "CLOSER: a collaborative locality-aware overlay SERvice," IEEE Transactions on Parallel and Distributed Systems, vol. 23, no. 6, pp. 1030-1037, 2012.

[9] S. Ren, E. Tan, T. Luo, S. Chen, L. Guo, and X. Zhang, “TopBT: a topology-aware and infrastructure-independent BitTorrent client," in Proceedings of the 29th IEEE International Conference on Computer Communications (INFOCOM '10), San Diego, Calif, USA, March 2010.
[10] S. Raza, G. Huang, C. N. Chuah, S. Seetharaman, and J. P. Singh, "MeasuRouting: a framework for routing assisted traffic monitoring," IEEE/ACM Transactions on Networking, vol. 20, no. 1, pp. 45-56, 2012.

[11] A. R. Sharafat, S. Das, G. Parulkar, and N. McKeown, "MPLSTE and MPLS VPNs with OpenFlow," in Proceedings of the ACM SIGCOMM 2011 Conference, pp. 452-453, Ontario, Canada, August 2011.

[12] S. Sundaresan, C. Lumezanu, N. Feamster, and P. Francois, "Autonomous traffic engineering with self-configuring topologies," in Proceedings of the 7th International Conference on Autonomic Computing (SIGCOMM '10), pp. 417-418, New Delhi, India, September 2010.

[13] N. Wang, K. H. Ho, G. Pavlou, and M. Howarth, "An overview of routing optimization for internet traffic engineering," IEEE Communications Surveys and Tutorials, vol. 10, no. 1, pp. 36-56, 2008.

[14] V. Aggarwal, A. Feldmann, and C. Scheideler, "Can ISPs and P2P users cooperate for improved performance?" ACM Computer Communication Review, vol. 37, no. 3, pp. 29-40, 2007.

[15] H. Xie, Y. R. Yang, A. Krishnamurthy, Y. G. Liu, and A. Silberschatz, "P4p: provider portal for applications," in Proceedings of the ACM SIGCOMM 2008 Conference on Data Communication, pp. 351-362, Seattle, Wash, USA, August 2008.

[16] W. Jiang, Z. S. Rui, J. Rexford, and M. Chiang, "Cooperative content distribution and traffic engineering in an ISP network," in Proceedings of the 11th International Joint Conference on Measurement and Modeling of Computer Systems (SIGMETRICS '09), pp. 239-250, Seattle, Wash, USA, June 2009.

[17] H. Lee, A. Nakao, and J. Kim, "BiCo: network operator-friendly P2P traffic control through bilateral cooperation with peers," Computer Networks, vol. 55, no. 9, pp. 2023-2034, 2011.

[18] G. Dán, T. Hoßfeld, S. Oechsner et al., "Interaction patterns between P2P content distribution systems and ISPs," IEEE Communications Magazine, vol. 49, no. 5, pp. 222-230, 2011.

[19] C. Xu, T. Liu, J. Guan, H. Zhang, and G. M. Muntean, "CMTQA: quality-aware adaptive concurrent multipath data transfer in heterogeneous wireless networks," IEEE Transactions on Mobile Computing, vol. 12, no. 11, pp. 2193-2205, 2013.

[20] C. Xu, F. Zhao, J. Guan, H. Zhang, and G. M. Muntean, "QoE-Driven user-Centric VoD services in urban multihomed P2P-based vehicular networks," IEEE Transactions on Vehicular Technology, vol. 62, no. 5, pp. 2273-2289, 2013.

[21] J. F. Nash, "Non-cooperative games," Annals of Mathematics, vol. 54, pp. 286-295, 1951.

[22] R. Selten, "Reexamination of the perfectness concept for equilibrium points in extensive games," International Journal of Game Theory, vol. 4, no. 1-2, pp. 25-55, 1975.

[23] H. Van Stackelberg, D. Bazin, R. Hill, and L. Urch, Market Structure and Equilibrium, Springer, 2011.

[24] M. J. Osborne, An Introduction to Game Theory, Oxford University Press, 2004.

[25] N. Wang, K. H. Ho, and G. Pavlou, "AMPLE: an adaptive traffic engineering system based on virtual routing topologies," IEEE Communications Magazine, vol. 50, no. 3, pp. 185-191, 2012.

[26] Abilene, http://www.internet2.edu.

[27] GT-ITM's Web Site, http://www.cc.gatech.edu/projects/gtitm/.

[28] H. Zheng, E. K. Lua, M. Pias, and T. G. Griffin, "Internet routing policies and round-trip-times," Passive and Active Network Measurement, vol. 3431, pp. 236-250, 2005. 
[29] Y. Zhang, "Abilene traffic matrix," http://www.cs.utexas.edu/ yzhang/research/AbileneTM/.

[30] J. S. Otto, M. A. Sánchez, D. R. Choffnes, F. E. Bustamante, and G. Siganos, "On blind mice and the elephant: understanding the network impact of a large distributed system," in Proceedings of the ACM SIGCOMM 2011 Conference, pp. 110-121, Ontario, Canada, August 2011. 


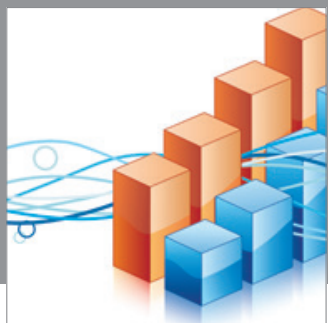

Advances in

Operations Research

mansans

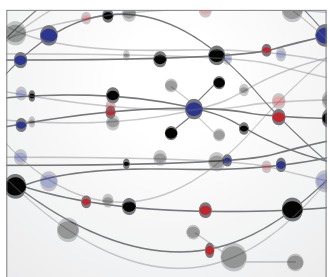

The Scientific World Journal
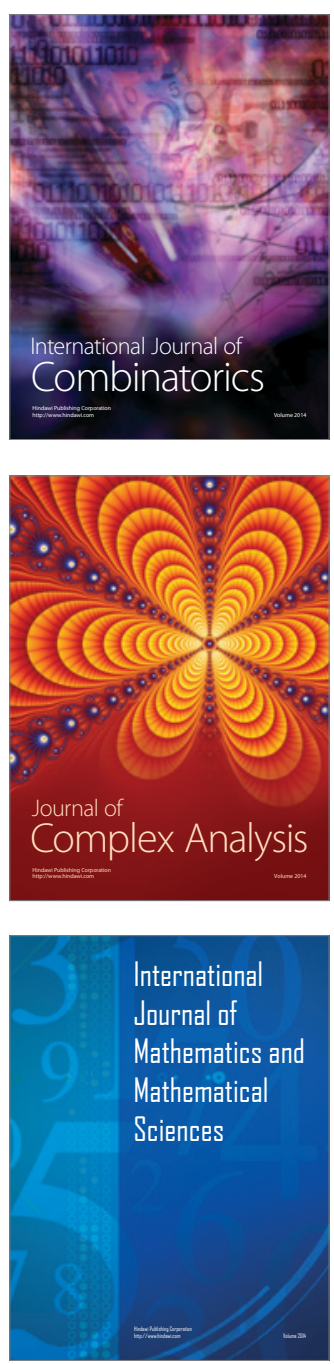
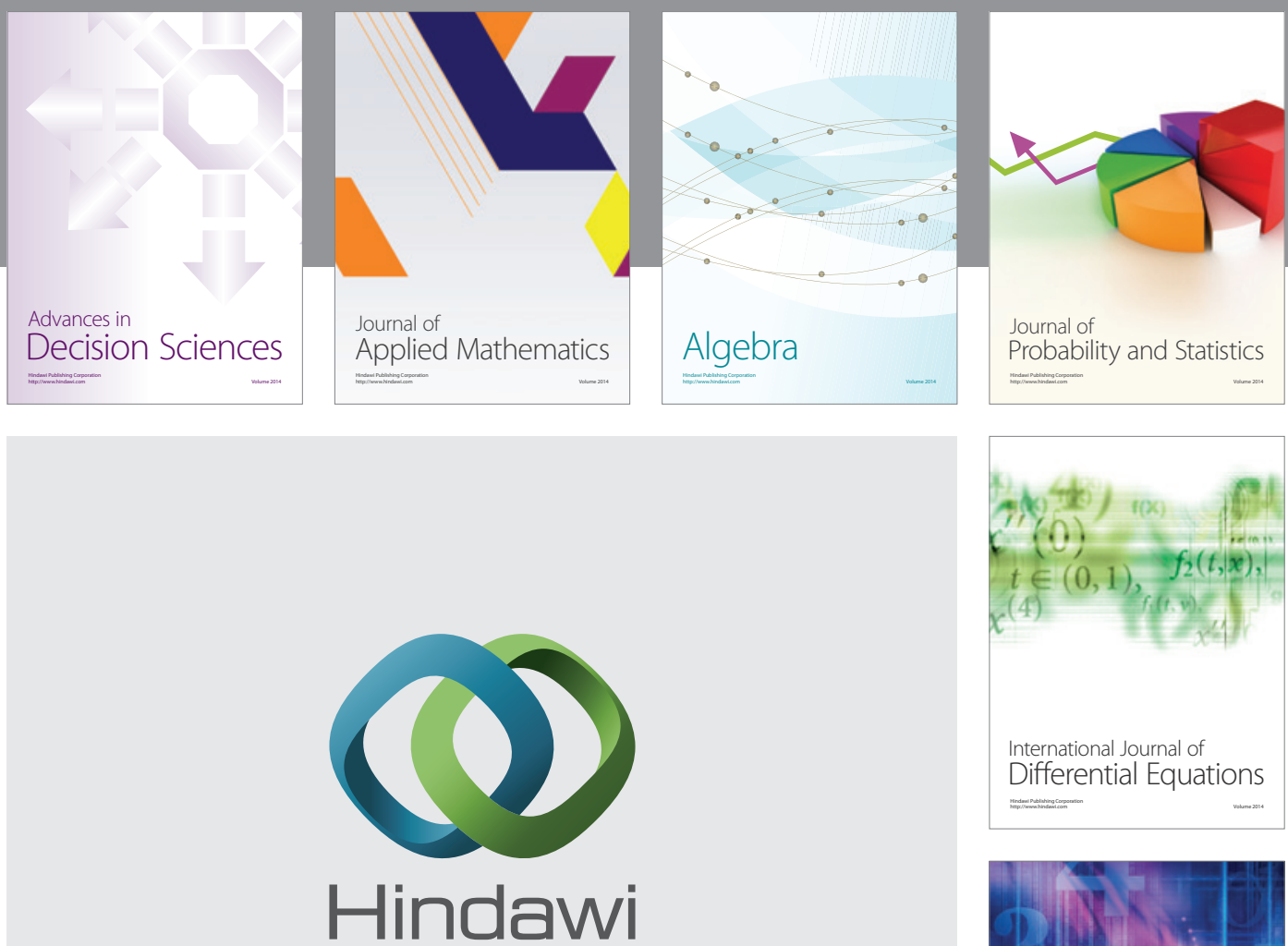

Submit your manuscripts at http://www.hindawi.com
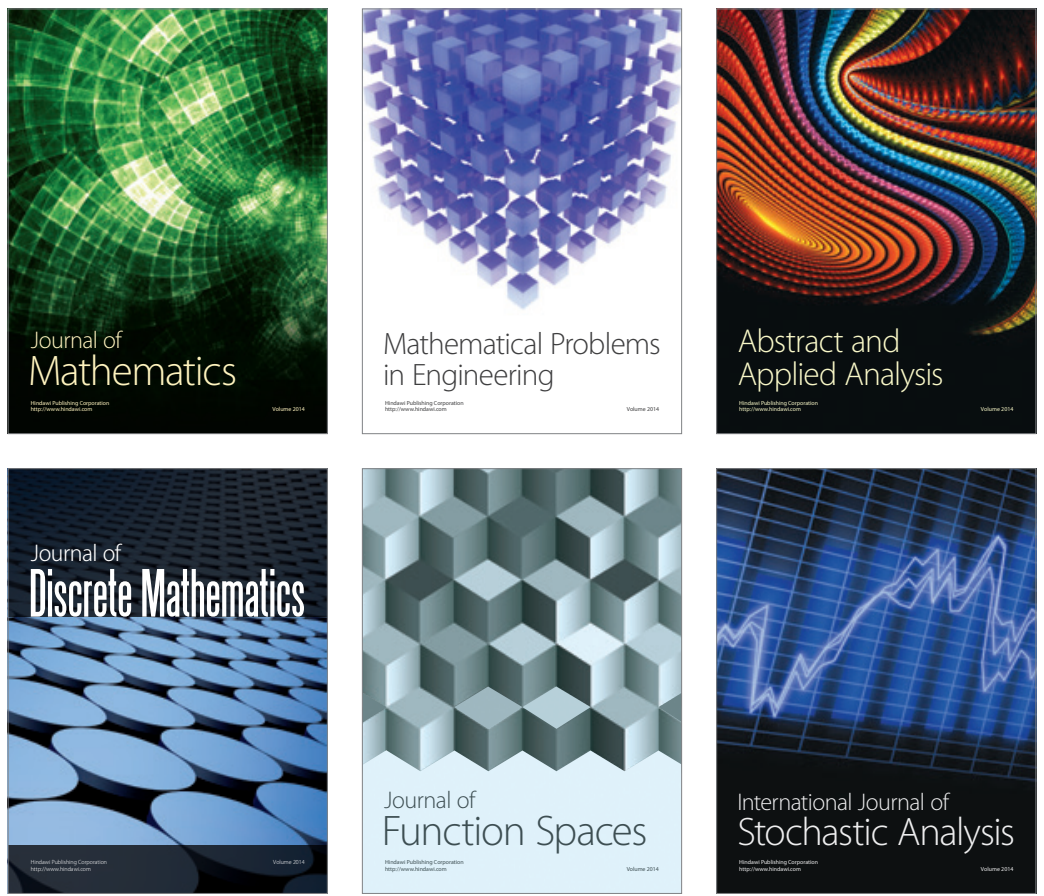

Journal of

Function Spaces

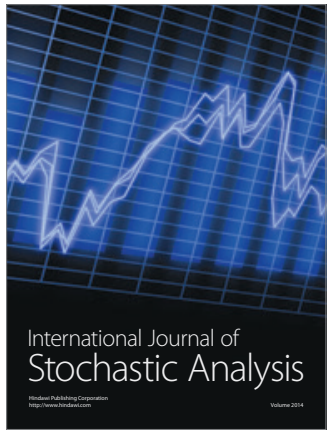

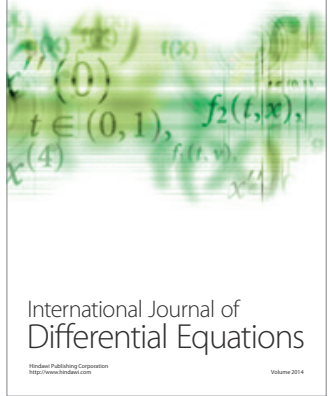
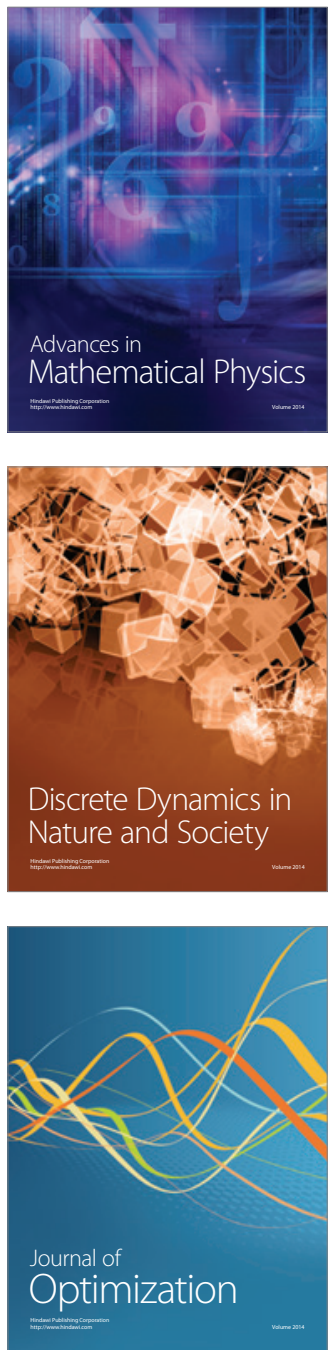